\title{
Mycorrhizal efficiency in pepper yield by fertilization in clay soil growth conditions
}

\author{
Hüseyin Karaca \\ Mustafa Kemal University, Agricultural Faculty, Soil Science and Plant Nutrition Department, 31034 Antakya, \\ Hatay, Turkey \\ *Corresponding author: hkaraca@mku.edu.tr
}

\begin{abstract}
Biomass responses to mycorrhizae and fertilization of phosphorus (P) and elemental sulfur (ES) on green pepper (Capsicum аппиum L.) grown for 45 days on calcareous sterilized Menekşe soil (sub-group Typic Xerorthent) were investigated. Root yield was increased by mycorrhizal inoculation compared to the control treatment. However, shoot yield remained unchanged. On the other hand, there was more synergistic effect between mycorrhizae and combined fertilization of ES and P, compared to the ES or P fertilization alone. Accordingly, shoot concentrations of $\mathrm{P}$ significantly increased. The other shoot nutrient concentrations differed independently from each other as statistically significant. Results showed that P and ES fertilization increased the efficieny of mycorrhizae in the clay soil growth conditions and mycorrhizae has potential to increase yield.
\end{abstract}

Key words: Mycorrhizae; elemental sulfur; phosphorus; shoot nutrient concentrations; pepper

\section{INTRODUCTION}

Mycorrhizae as a kind of substitute of plant root in the nutrient uptake for plant growth has different interactions in changing growth medium conditions, resulting in the differences in the productivity of natural plant production system. Understanding of those interactions may clarify mycorrhizal benefits to ecological agriculture. Mycorrhizal inoculation increases yield (Karaca, 2012a; Karaca, 2013; Karaca et al., 2013). Similarly, P fertilization results in significant yield increases. On the other hand, in clay textured soil, $\mathrm{P}$ and mycorrhizae addition in combination compared to $\mathrm{P}$ addition alone results in significant shoot yield decrease while the reverse is the case for the root yield. (Karaca et al., 2013). Both mycorrhizal inoculation and its effect by $\mathrm{P}$ applications increases yield (Ortas et al., 1996; Karaca, 2012a).

Mycorrhizal inoculation increases yield but S fertilization has no effect on the yield (Guo et al., 2005). ES fertilization affects the yield in both directions (Karaca, 2012b). S fertilization has no effect (Hoffman et al., 1998) or increases yield (McLaughlin and Holford 1982; Merrien, 1987). Oxidation products of ES decreases soil $\mathrm{pH}$ that gradually increases solubility of plant nutrients. Heavy metal concentration, if present, can increase by the decreased soil $\mathrm{pH}$ resulting in yield decrease (Cui et al., 2004).

Mycorrhizal inoculation induces higher shoot and root yield. Root to shoot ratio decreases or remains around at the same level while shoot $\mathrm{P}$ concentration increases or remains around at the same level depending on the mycorrhizal species (Ortas et al., 2002). Mycorrhizal inoculation alone compared to 
the control treatment results in increased or unchanged root to shoot ratio. However, those ratios are in both directions in the case of ES and/or P additions. (Karaca, 2012a). Romero et al., (1996) proposed that there may be an optimum root to shoot ratio for plant growth.

Mycorrhizal inoculation alone compared to the control treatment decreases the shoot $\mathrm{P}$ concentrations (Karaca, 2012a). There is no correlation, all the time, between increased $\mathrm{P}$ uptake and $\mathrm{P}$ concentration in plant dry matter (Menge et al., 1978; Raj et al., 1981; Yibirin et al., 1996; Karaca, 2012a; Karaca, 2012b; Karaca et al., 2013). With small additions of P fertilizer, entry points and fungal growth on the root surface remains normal but arbuscles are small and even fewer in number, reducing the effectiveness of fungus/plant relationship. Mycorrhizal infections tend to stop in soils containing or given high P (Baylis, 1967; Mosse, 1967; Karaca, 2012a). ES fertilizations does not affect the root mycorrhizal infection level but can compensate the decreasing effect of $\mathrm{P}$ fertilization on the root mycorrhizal infection level and can increase efficient work of mycorrhizae for the yield (Karaca, 2012a). A slight reduction on percentage of mycorrhizal colonization was noted with $\mathrm{SO}_{2}$ (Diaz et al., 1996).

This study evaluates the effects of ES and/or $\mathrm{P}$ on mycorrhizae for the yield, shoot nutrient concentrations, and changes in the root morphology of pepper in clay soil growth conditions.

\section{MATERIAL AND METHOD}

Surface soil samples $(0-30 \mathrm{~cm})$ for Menekşe soil were taken from the non cultivated part of the Cukurova University experimental farm. The soil Menekşe serial was a typic Xerorthent of the Entisol ordo in the Soil Taxonomy (Özbek et al., 1974). The plot had not been cultivated for many years. Air dried soil samples were crushed, sieved $\left(2 \mathrm{~mm}\right.$ mesh opening) and autoclaved at $121^{\circ} \mathrm{C}$ for two hours prior to use as a growth medium. The pots surface were sterilized with ethanol $96 \%(\mathrm{v} / \mathrm{v})$, washed by distilled water and dried out prior to the use. $4 \mathrm{~kg}$ of autoclaved soil were placed in the plastic pots and following treatments were made.

MoPoSo: Control application in which $500 \mathrm{mg} \mathrm{kg}^{-1} \mathrm{~N}$ (as urea), $250 \mathrm{mg} \mathrm{kg}^{-1} \mathrm{~K}$ (as $\mathrm{KNO}_{3}$ ), $5 \mathrm{mg} \mathrm{kg}^{-1}$ $\mathrm{Zn}$ (as $\mathrm{ZnSO}_{4}$ ) and $20 \mathrm{mg} \mathrm{kg}^{-1} \mathrm{Fe}$ (as Fe-EDDHA) were put into the pots, and then soil samples were thoroughly mixed.

MoPoS+: $100 \mathrm{mg} \mathrm{kg}^{-1} \mathrm{ES}$ was added to the control treatment.

MoP+So: $100 \mathrm{mg} \mathrm{kg}^{-1} \mathrm{P}$ (as triple super phosphate) was added to the control treatment.

$\mathbf{M o P}+\mathbf{S}+: 100 \mathrm{mg} \mathrm{kg}^{-1} \mathrm{P}$ and $100 \mathrm{mg} \mathrm{kg}^{-1} \mathrm{ES}$ were added to the control treatment.

M+PoSo: Glomus mossea AM fungi type as the mycorrhizae (as $145 \mathrm{~g}$ soil taken from the vicinity of the dead vineyard roots at the University Farm for the average 1000 spore/pot inoculation) was added to the control treatment. The mycorrhizal density of soil was determined by the method of Gerdemann and Nicolson (1963).

M+PoS+: The mycorrhizae and $100 \mathrm{mg} \mathrm{kg}^{-1}$ ES were added to the control treatment.

$\mathbf{M}+\mathbf{P}+$ So: The mycorrhizae and $100 \mathrm{mg} \mathrm{kg}^{-1} \mathrm{P}$ were added to the control treatment.

$\mathbf{M}+\mathbf{P}+\mathbf{S}+$ : The mycorrhizae, $100 \mathrm{mg} \mathrm{kg}^{-1} \mathrm{P}$ and $100 \mathrm{mg} \mathrm{kg}^{-1} \mathrm{ES}$ were added to the control treatment. All fertilizers were mixed thoroughly in the soil. However, the mycorrhizal inoculum was mixed into the top $5 \mathrm{~cm}$ of the soil. Following the addition of the inoculum, $1000 \mathrm{ml}$ water was added to the each pot to bring the soil about field capacity and allowed to drain for 5 days.

Green pepper seeds (Capsicum annuиmL.) were sown into sterilized growth medium of soil and organic matter mixture (soil/organic matter: $2 / 1(\mathrm{v} / \mathrm{v})$ and grown for 35 days. The seedlings were carefully extracted from the nursery and transplanted into the pots and irrigated when required. The seedlings grew for one and half month. The plants were harvested by cutting just above the soil level and the shoots were separately dried at $75^{\circ} \mathrm{C}$ to a constant weight after clearing possible contaminants by tap water and then distilled water. Plants samples were dried to constant weight at $75^{\circ} \mathrm{C}$ and their particle size were heavy clay below $0.5 \mathrm{~mm}$ to obtain homogenous samples. 
$\mathrm{N}$ (nitrogen) content of samples was determined by Kjeldahl digestion and steam distillation (Lees 1971). For determination of other nutrient elements samples were digested in $\mathrm{HNO}_{3}$ and $\mathrm{H}_{2} \mathrm{O}_{2}(\mathrm{v} / \mathrm{v}$ : 4/1) mixture (Cem, MarsXpress Manual). P content of the digests were colorimetrically determined (Shimadzu 1201 model UV/VIS spectrometer) according to Murphy and Riley (1962) and K (potassium), $\mathrm{Na}$ (sodium), $\mathrm{Ca}$ (calcium), $\mathrm{Mg}$ (magnesium), $\mathrm{Fe}$ (iron), $\mathrm{Cu}$ (copper), $\mathrm{Mn}$ (manganese) and $\mathrm{Zn}$ (zinc) contents were determined using ICP-OES (Varian, Liberty Series II) according to Kacar (1972).

After separating from the soil, the fresh roots were washed under running tap water, followed by distilled water and dried on tissue paper. Before drying, small root samples were preserved in a mixture (250:13:15) of ethanol, glacial acetic acid and formalin (Ortas et al., 2004) until the determination of mycorrhizal infection. The root clearing and staining procedure and the degree of mycorrhizal infection in the root cortex was assessed by the method of Koske and Gemma (1989). After sampling, the roots were dried and weighed for root biomass.

Basic physical and chemical properties of autoclaved soil were analyzed as follows: soil texture analysis by a hydrometer (Bouyoucos, 1951), organic matter by using Lichterfelder wet ashing (Schlichting and Blume, 1966), soil reaction and electrical conductivity by means of a combined electrode and EC meter in saturation paste, respectively (Schlichting and Blume 1966), Ca carbonate equivalent by a manometric method (Loeppert and Suarez, 1996), cation exchange capacity (CEC) by saturating sodium acetate $(1 \mathrm{M} \mathrm{pH} 8.2)$ and then replacing the $\mathrm{Na}$ with ammonium acetate $(1 \mathrm{M} \mathrm{pH}$ 7.0) (U.S. Salinity Laboratory Staff, 1954), available phosphorus by Olsen method (Olsen et al., 1954), total nitrogen (N) by Bremner (1996), soil nitrate by Fabig (1979), soil ammonium by Fachgruppe Wasserchemie in der Gesellscheft Deutscher Chemiker (1983), exchangeable potassium (K) with neutral ammonium acetate by Pratt and Morse (1954), DTPA extractable microelements (Fe, $\mathrm{Zn}, \mathrm{Cu}$ and $\mathrm{Mn}$ ) by Lindsay and Norvell (1978), soil density by a picnometer by Blake and Hartge (1986b), bulk density by Blake and Hartge (1986a) and permeability by a constant head permeameter by Klute and Dirksen (1986).

The Menekşe soil series are classified as a clay textured soil (sand $257 \mathrm{~g} \mathrm{~kg}^{-1}$; silt $84.8 \mathrm{~g} \mathrm{~kg}^{-1}$; clay $\left.658.2 \mathrm{~g} \mathrm{~kg}^{-1}\right)$. The $\mathrm{pH}$ of the soil is slightly alkaline (7.74) and there is a slight salinity problem (EC = $\left.4.76 \mathrm{dSm}^{-1}\right)$. The organic matter content is low $\left(5.13 \mathrm{~g} \mathrm{~kg}^{-1}\right)$, while the CEC is $36.31 \mathrm{cmol} \mathrm{kg}^{-1}$, density is $2.66 \mathrm{~g} \mathrm{~cm}^{-3}$; bulk density is $1.519 \mathrm{~g} \mathrm{~cm}^{-3}$; porosity $42.9 \%$, and the permeability is $1.84 \mathrm{~cm} \mathrm{~h}^{-}$ ${ }^{1}$ (medium-low). The plant nutrients of the soil are low: $\mathrm{C} 3.00 \mathrm{~g} \mathrm{~kg}^{-1} ; \mathrm{P} 3.93 \mathrm{mg} \mathrm{kg}^{-1} ; \mathrm{K}_{155} \mathrm{~m} \mathrm{~kg}^{-1}$; $\mathrm{NH}_{4} 3.64 \mathrm{mg} \mathrm{kg}^{-1} ; \mathrm{NO}_{3} 2.70 \mathrm{mg} \mathrm{kg}^{-1}$; total $\mathrm{N} 0.4 \mathrm{~g} \mathrm{~kg}^{-1} ; \mathrm{Fe} 0.305 \mathrm{mg} \mathrm{kg}^{-1} ; \mathrm{Cu} 0.11 \mathrm{mg} \mathrm{kg}^{-1} ; \mathrm{Mn}_{0.172}$ $\mathrm{mg} \mathrm{kg}^{-1}$ and $\mathrm{Zn} 0.082 \mathrm{mg} \mathrm{kg}^{-1}$. Soil is very calcareous with $470 \mathrm{~g} \mathrm{~kg}^{-1} \mathrm{CaCO}_{3}$ content.

The data were subjected to the analysis of variance using MSTAT-C statistical analysis package (MSTATC, Michigan State University, East Lansing, MI, USA). The mean separation was made by Least Significant Difference (LSD) test at $\mathrm{P}<0.05$. Root microphotographs were taken by the scanning electron microscope (Jeol JSM-5500LV).

\section{RESULTS}

Mycorrhizal inoculation and phosphorus and sulfur fertilizations significantly affected yield, mycorrhizal infection percent and nutrient uptake (Table 1).

\section{Shoot and Root Yield and, Shoot Nutrient Concentration Responses to ES, P and Mycorrhizal Inoculation}

Root yield by M+PoSo treatment compared to MoPoSo one significantly increased as shown in Figure 1 and, Table 2. Those results are in line with previous findings (Ortas et al., 1996; Ortas et al., 2002; 
Guo et al., 2005; Karaca, 2012a; Karaca, 2013; Karaca et al., 2013) but the shoot yield was unchanged (Figure 1, and, Table 2). Nevertheless, there were no correlations all the time between the yield and shoot nutrient concentrations. In that respect, higher yields compared to lower yields can show shoot nutrient concentrations in both ways for any nutrient independently from any other one (Figure 2, 3 and, Table 2). Those results are in line with the previous findings (Menge et al., 1978; Raj et al., 1981; Yibirin et al., 1996; Karaca, 2012a; Karaca, 2012b ; Karaca et al., 2013).

MoPoS + treatment compared to the MoPoSo one significantly decreased the shoot and root yield. The decrease of yield are not in parellel with the previous findings (McLaughlin and Holford 1982; Merrien 1987) but in accordance with the findings (Karaca, 2012b). Those yield decreases can be related to the shoot heavy metal concentrations. Thus, as presented in Figure 3, and Table 2, there were higher shoot $\mathrm{Fe}, \mathrm{Mn}, \mathrm{Zn}$ concentrations for the MoPoS+ treatment compared to the MoPoSo treatment in the non-mycorrhizal treatments. Accordingly, there may be mimic of heavy metal poisoning as is in the previous findings (Cui et al., 2004) who reported that sulfur application can increase the solubility of the nutrients for the plant uptake to result in higher growth but, in the case of heavy metal presence, the increased heavy metal in the growth conditions can cause poisining effect of heavy metals resulting in yield decrease.

$\mathrm{M}+\mathrm{PoS}+$ treatment compared to the $\mathrm{M}+\mathrm{PoS}$ one resulted in root yield decrease and no shoot yield difference. The root yield decrease and the indifference in the shoot yield are similar to the previous findings (Karaca, 2012b). The root yield decrease can be attributed to the differences in the shoot heavy metal concentrations. Thus, M+PoS+ treatment compared to M+PoSo one in the mycorrhizal treatments had higher shoot Fe concentration, but lower shoot $\mathrm{Zn}$ and Mn concentrations (Table 2). However, the unchanged shoot yield by $\mathrm{M}+\mathrm{PoS}+$ treatment compared to the M+PoSo one is similar to the previous findings (Hoffman et al., 1998; Guo et al., 2005).

Table 1. Analysis of variance for shoot and root yield, mycorrhizal infection and nutrient uptake in Menekșe soil.

\begin{tabular}{|c|c|c|c|c|c|c|c|}
\hline \multirow[b]{2}{*}{ Variation source } & \multirow[b]{2}{*}{ D.F. } & \multicolumn{2}{|c|}{ Shoot Dry Weight } & \multicolumn{2}{|c|}{ Root Dry Weight } & \multicolumn{2}{|c|}{ Root:Shoot Ratio } \\
\hline & & Mean square & $\begin{array}{l}\text { F value } \\
\text { (Prob.) }\end{array}$ & $\begin{array}{l}\text { Mean } \\
\text { square }\end{array}$ & $\begin{array}{l}\text { F value } \\
\text { (Prob.) }\end{array}$ & $\begin{array}{c}\text { Mean } \\
\text { square }\end{array}$ & $\begin{array}{l}\text { F value } \\
\text { (Prob.) }\end{array}$ \\
\hline Replicate & 2 & 1757.292 & $\begin{array}{c}1.9471 \\
(0.1794)\end{array}$ & 1.042 & 0.1063 & 0 & 0.1338 \\
\hline Mycorrhizae(M) & 1 & 808501.042 & $\begin{array}{c}895.8165 \\
(<0.0001) \\
41.2279\end{array}$ & 9009.375 & $\begin{array}{c}918.9891 \\
(<0.0001) \\
112.9223\end{array}$ & $\begin{array}{l}0.001 \\
0\end{array}$ & $\begin{array}{l}100.4562 \\
(<0.0001) \\
5.2476\end{array}$ \\
\hline Sulfur(S) & 1 & 37209.375 & $\begin{array}{l}(<0.0001) \\
5103998\end{array}$ & 1107.042 & $\begin{array}{c}(<0.0001) \\
1361791\end{array}$ & & $\begin{array}{l}(0.038) \\
13.3792\end{array}$ \\
\hline $\mathrm{MxS}$ & 1 & 460651.042 & $\begin{array}{c}(<0.0001) \\
138091949\end{array}$ & 1335.042 & $\begin{array}{l}(<0.0001) \\
60896557\end{array}$ & 0 & $\begin{array}{l}13.3 / 92 \\
(0.0026) \\
22.2316\end{array}$ \\
\hline Phosphorus(P) & 1 & 12463209.38 & $(<0.0001)$ & 59700.375 & $\begin{array}{c}(<0.0001) \\
482.6855\end{array}$ & . & $(0.0003)$ \\
\hline $\mathrm{MxP}$ & 1 & 556626.042 & $\begin{array}{c}616.7398 \\
(<0.0001) \\
148.7497\end{array}$ & 4732.042 & $\begin{array}{c}482.6855 \\
(<0.0001) \\
226.7923\end{array}$ & 0 & $\begin{array}{l}(0.4366 \\
(0.006) \\
14.7403\end{array}$ \\
\hline SxP & 1 & 134251.042 & $\begin{array}{c}(<0.0001) \\
306.1129\end{array}$ & 2223.375 & $\begin{array}{c}(<0.0001) \\
91.8415\end{array}$ & 0 & $\begin{array}{l}(0.0018) \\
0.3771\end{array}$ \\
\hline $\begin{array}{c}\text { MxSxP } \\
\text { Error }\end{array}$ & $\begin{array}{c}1 \\
14\end{array}$ & $\begin{array}{c}276276.042 \\
902.53\end{array}$ & $(<0.0001)$ & $\begin{array}{l}900.375 \\
9.804\end{array}$ & $(<0.0001)$ & 0 & \\
\hline $\begin{array}{l}\text { Coefficient of } \\
\text { Variation }(\%)\end{array}$ & & 3 & & & & & 21 \\
\hline
\end{tabular}


Continued Table 1.

\begin{tabular}{|c|c|c|c|c|c|c|c|}
\hline \multirow[b]{2}{*}{ Variation source } & \multirow[b]{2}{*}{ D.F. } & \multicolumn{2}{|c|}{ Mycorrhizal Infection } & \multicolumn{2}{|c|}{ Nitrogen Uptake } & \multicolumn{2}{|c|}{ Phosphorus Uptake } \\
\hline & & $\begin{array}{l}\text { Mean } \\
\text { square }\end{array}$ & $\begin{array}{l}\text { F value } \\
\text { (Prob.) }\end{array}$ & $\begin{array}{l}\text { Mean } \\
\text { square }\end{array}$ & $\begin{array}{l}\text { F value } \\
\text { (Prob.) }\end{array}$ & Mean square & $\begin{array}{l}\text { F value } \\
\text { (Prob.) }\end{array}$ \\
\hline Replicate & 2 & 16.667 & $\begin{array}{c}1 \\
420.25\end{array}$ & 18954.167 & $\begin{array}{c}0.0204 \\
302.063\end{array}$ & $\begin{array}{l}3457.292 \\
281666.667\end{array}$ & $\begin{array}{l}1.6353 \\
(0.2357) \\
130.7815\end{array}$ \\
\hline Mycorrhizae(M) & 1 & 7004.167 & $\begin{array}{c}(<0.0001) \\
12.25\end{array}$ & 280576817 & $\begin{array}{c}(<0.0001) \\
11.9182\end{array}$ & 201000.001 & $\begin{array}{l}(<0.0001) \\
0\end{array}$ \\
\hline Sulfur(S) & 1 & 204.167 & $\begin{array}{c}(0.0035) \\
12.25\end{array}$ & 11070416.7 & $\begin{array}{c}(0.0039) \\
40.8035\end{array}$ & 375000 & 174.1173 \\
\hline $\mathrm{MxS}$ & 1 & 204.167 & $(0.0035)$ & 37901066.7 & $(<0.0001)$ & & $(<0.0001)$ \\
\hline Phosphorus(P) & 1 & 704.167 & $\begin{array}{c}42.25 \\
(<0.0001) \\
42.25\end{array}$ & 44390400 & $\begin{array}{c}47.7898 \\
(<0.0001) \\
38.6149\end{array}$ & $\begin{array}{l}4166666.667 \\
1666.667\end{array}$ & $\begin{array}{l}1934.6369 \\
(<0.0001) \\
0.7739\end{array}$ \\
\hline $\mathrm{MxP}$ & 1 & 704.167 & $(<0.0001)$ & 35868150 & $(<0.0001)$ & & \\
\hline $\mathrm{SxP}$ & 1 & 104.167 & $\begin{array}{c}6.25 \\
(0.0255) \\
6.25\end{array}$ & 1892816.67 & $\begin{array}{c}2.0378 \\
(0.1754) \\
65.7322\end{array}$ & 26666.667 & $\begin{array}{l}12.3817 \\
(0.0034) \\
341.27\end{array}$ \\
\hline MxSxP & 1 & 104.167 & $(0.0255)$ & 61056600 & $(<0.0001)$ & & $(<0.0001)$ \\
\hline Error & 14 & 16.667 & & 928868.452 & & 2153.72 & \\
\hline $\begin{array}{l}\text { Coefficient of } \\
\text { Variation }(\%)\end{array}$ & & 23 & & 2.0 & & 2.1 & \\
\hline \multicolumn{8}{|l|}{ Continued Table 1.} \\
\hline & & \multicolumn{2}{|c|}{ Potassium Uptake } & \multicolumn{2}{|c|}{ Calcium Uptake } & \multicolumn{2}{|c|}{ Magnesium Uptake } \\
\hline Variation source & D.F. & $\begin{array}{l}\text { Mean } \\
\text { square }\end{array}$ & $\begin{array}{l}\text { F value } \\
\text { (Prob.) }\end{array}$ & $\begin{array}{l}\text { Mean } \\
\text { square }\end{array}$ & $\begin{array}{l}\text { F value } \\
\text { (Prob.) }\end{array}$ & Mean square & $\begin{array}{l}\text { F value } \\
\text { (Prob.) }\end{array}$ \\
\hline \multirow[t]{2}{*}{ Replicate } & 2 & 26852.478 & 0.1226 & 21625.452 & 0.6561 & 9711.354 & 0.3387 \\
\hline & & & 47.3115 & 3674385.81 & 111.4737 & & 19.9455 \\
\hline \multirow[t]{2}{*}{ Mycorrhizae(M) } & 1 & 10359446.4 & $(<0.0001)$ & & $(<0.0001)$ & 571836.063 & $(0.0005)$ \\
\hline & & & 28.3411 & 26606.772 & 0.8072 & & 22.5471 \\
\hline \multirow[t]{2}{*}{ Sulfur(S) } & 1 & 6205631.71 & $(0.0001)$ & & & 646422.663 & $(0.0003)$ \\
\hline & & & 25.2238 & 2401918.7 & 72.8695 & & 1.3133 \\
\hline \multirow[t]{2}{*}{$\mathrm{MxS}$} & 1 & 5523059.12 & $(0.0002)$ & & $(<0.0001)$ & 37651.728 & $(0.271)$ \\
\hline & & & 227.3984 & 2155622.63 & 65.3974 & & 94.0714 \\
\hline \multirow[t]{2}{*}{ Phosphorus(P) } & 1 & 49791696 & $(<0.0001)$ & & $(<0.0001)$ & 2697018.822 & $(<0.0001)$ \\
\hline & & & 2.7926 & 538950.364 & 16.3507 & & 1.0441 \\
\hline \multirow[t]{2}{*}{$\mathrm{MxP}$} & 1 & 611477.546 & $(0.1169)$ & & $(0.0012)$ & 29934.448 & $(0.3242)$ \\
\hline & & & 21.2929 & 1039542.71 & 31.5377 & & \\
\hline \multirow[t]{2}{*}{$\mathrm{SxP}$} & 1 & 4662343.89 & $(0.0004)$ & & $(0.0001)$ & 19482.591 & 0.6795 \\
\hline & & & & 14974.986 & 0.4543 & & 12.521 \\
\hline $\mathrm{MxSxP}$ & 1 & 26710.76 & 0.122 & & & 358975.008 & $(0.0033)$ \\
\hline Error & 14 & 218962.341 & & 32961.909 & & 28669.917 & \\
\hline $\begin{array}{l}\text { Coefficient of } \\
\text { Variation }(\%)\end{array}$ & & 2 & 54 & & & 2 & \\
\hline
\end{tabular}

$\mathrm{MoP}+\mathrm{So}$ treatment compared to MoPoSo treatment significantly increased the shoot and root yield in the non-mycorrhizal treatments (Table 2) being in line with the findings (Ortas et al., 1996; Karaca, 2012a). Those yield increases can be related to the low P content of soil. So, it could be expected that $\mathrm{P}$ fertilization in soils low in $\mathrm{P}$ content can increase the yield. Interestingly, the increase in question in both shoot and root yield by $\mathrm{M}+\mathrm{P}+\mathrm{So}$ treatment in the mycorrhizal treatments compared to the $\mathrm{MoP}+\mathrm{So}$ one in the non-mycorrhizal treatments was higher indicating the synergism between mycorrhizae and P being not consistent with the findings (Baylis, 1967; Mosse, 1967; Karaca et al. 2013). 
Continued Table 1.

\begin{tabular}{|c|c|c|c|c|c|c|c|}
\hline \multirow[b]{2}{*}{ Variation source } & \multirow[b]{2}{*}{ D.F. } & \multicolumn{2}{|c|}{ Iron Uptake } & \multicolumn{2}{|c|}{ Zinc Uptake } & \multicolumn{2}{|c|}{ Copper Uptake } \\
\hline & & Mean square & $\begin{array}{l}\text { F value } \\
\text { (Prob.) }\end{array}$ & $\begin{array}{l}\text { Mean } \\
\text { square }\end{array}$ & $\begin{array}{l}\text { F value } \\
\text { (Prob.) }\end{array}$ & $\begin{array}{l}\text { Mean } \\
\text { square }\end{array}$ & $\begin{array}{l}\text { F value } \\
\text { (Prob.) }\end{array}$ \\
\hline Replicate & 2 & 49.115 & $\begin{array}{c}2.5174 \\
(0.1164) \\
182.0554\end{array}$ & 0.022 & $\begin{array}{l}0.0034 \\
2.0015\end{array}$ & 0.167 & $\begin{array}{c}1.3207 \\
(0.2982) \\
244.4604\end{array}$ \\
\hline Mycorrhizae(M) & 1 & 3551.938 & $\begin{array}{c}(<0.0001) \\
29.2897\end{array}$ & 13.261 & $\begin{array}{c}(0.179) \\
37.597\end{array}$ & 30.917 & $\begin{array}{c}(<0.0001) \\
37.5782\end{array}$ \\
\hline Sulfur(S) & 1 & 571.448 & $\begin{array}{c}(0.0001) \\
3.1376\end{array}$ & 249.099 & $\begin{array}{c}(<0.0001) \\
11.4449\end{array}$ & 4.753 & $\begin{array}{c}(<0.0001) \\
151.7237\end{array}$ \\
\hline $\mathrm{MxS}$ & 1 & 61.216 & $(0.0983)$ & 75.828 & $\begin{array}{c}(0.0045) \\
240.7046\end{array}$ & 19.189 & $\begin{array}{c}(<0.0001) \\
1825.605\end{array}$ \\
\hline Phosphorus(P) & 1 & 11.551 & 0.592 & 1594.792 & $\begin{array}{c}(<0.0001) \\
7.3127\end{array}$ & 230.888 & $(<0.0001)$ \\
\hline $\mathrm{MxP}$ & 1 & 0.377 & $\begin{array}{l}0.0193 \\
13.576\end{array}$ & 48.45 & $\begin{array}{c}(0.0171) \\
3.9873\end{array}$ & 0.047 & $\begin{array}{c}0.3702 \\
128.8982\end{array}$ \\
\hline $\mathrm{SxP}$ & 1 & 264.87 & $\begin{array}{l}(0.0025) \\
19.4327\end{array}$ & 26.418 & $\begin{array}{l}(0.0657) \\
101.4963\end{array}$ & 16.302 & $\begin{array}{c}(<0.0001) \\
11.8603\end{array}$ \\
\hline $\mathrm{MxSxP}$ & 1 & 379.135 & $(0.0006)$ & 672.465 & $(<0.0001)$ & 1.5 & $(0.004)$ \\
\hline Error & 14 & 19.51 & & 6.626 & & 0.126 & \\
\hline $\begin{array}{l}\text { Coefficient of } \\
\text { Variation }(\%)\end{array}$ & & 2.39 & & & & & \\
\hline
\end{tabular}

Continued Table 1.

\begin{tabular}{|c|c|c|c|c|c|}
\hline \multirow[b]{2}{*}{ Variation source } & \multirow[b]{2}{*}{ D.F. } & \multicolumn{2}{|c|}{ Manganese Uptake } & \multicolumn{2}{|c|}{ Sodium Uptake } \\
\hline & & $\begin{array}{l}\text { Mean } \\
\text { square }\end{array}$ & $\begin{array}{l}\text { F value } \\
\text { (Prob.) }\end{array}$ & Mean square & $\begin{array}{l}\text { F value } \\
\text { (Prob.) }\end{array}$ \\
\hline \multirow{3}{*}{ Replicate } & \multirow{3}{*}{2} & 24.931 & 1.8238 & & \\
\hline & & & $(0.1977)$ & 1284.838 & 0.1587 \\
\hline & & 18122.512 & 1325.7056 & & 67.0398 \\
\hline \multirow[t]{2}{*}{ Mycorrhizae(M) } & \multirow[t]{2}{*}{1} & & $(<0.0001)$ & 542589.095 & $(<0.0001)$ \\
\hline & & 304.451 & 22.2714 & & 14.3941 \\
\hline \multirow[t]{2}{*}{ Sulfur(S) } & \multirow[t]{2}{*}{1} & & $(0.0003)$ & 116499.421 & $(0.002)$ \\
\hline & & 1944 & 142.2083 & & 55.2629 \\
\hline \multirow[t]{2}{*}{$\mathrm{MxS}$} & \multirow[t]{2}{*}{1} & & $(<0.0001)$ & 447272.348 & $(<0.0001)$ \\
\hline & & 3804.194 & 278.286 & & 471.2597 \\
\hline \multirow[t]{2}{*}{ Phosphorus(P) } & \multirow[t]{2}{*}{1} & & $(<0.0001)$ & 3814155.254 & $(<0.0001)$ \\
\hline & & 4082.042 & 298.6112 & & \\
\hline \multirow[t]{2}{*}{$\mathrm{MxP}$} & \multirow[t]{2}{*}{1} & & $(<0.0001)$ & 6301.152 & 0.7785 \\
\hline & & 347.321 & 25.4073 & & 25.1072 \\
\hline \multirow[t]{2}{*}{$\mathrm{SxP}$} & \multirow[t]{2}{*}{1} & & $(0.0002)$ & 203205.882 & $(0.0002)$ \\
\hline & & 944.764 & 69.1118 & & 1.9911 \\
\hline $\mathrm{MxSxP}$ & 1 & & $(<0.0001)$ & 16114.947 & $(0.1801)$ \\
\hline Error & 14 & 13.67 & & 8093.532 & \\
\hline $\begin{array}{l}\text { Coefficient of } \\
\text { Variation }(\%)\end{array}$ & & \multicolumn{2}{|c|}{3.26} & \multicolumn{2}{|l|}{3.63} \\
\hline
\end{tabular}


Table 2. Response of pepper to mycorrhizal inoculation, fertilization with elemental sulfur and phosphorus in Menekşe soil.

\begin{tabular}{|c|c|c|c|c|c|c|c|}
\hline Treatment & Shoot DW (mg) & $\begin{array}{l}\text { Root DW } \\
(\mathrm{mg})\end{array}$ & $\mathrm{R} / \mathrm{S}$ & $\begin{array}{l}\quad \begin{array}{l}\text { Menekşe } \\
\text { soil } \\
\text { Mycorrhizal } \\
\text { infection }(\%)\end{array} \\
\end{array}$ & $\begin{array}{l}\text { N content } \\
(\mathrm{mg} / \mathrm{kg})\end{array}$ & $\begin{array}{l}\text { P content } \\
(\mathrm{mg} / \mathrm{kg})\end{array}$ & $\begin{array}{l}\text { K content } \\
(\mathrm{mg} / \mathrm{kg})\end{array}$ \\
\hline MoPoSo & $315.00 \mathrm{e}$ & $20.67 f$ & 0.07 & $0.00 \mathrm{~d}$ & $49606.67 \mathrm{c}$ & $1566.67 \mathrm{~g}$ & 16052.81 \\
\hline $\mathrm{MoP}+\mathrm{So}$ & $1516.67 \mathrm{c}$ & $85.33 d$ & 0.06 & $0.00 \mathrm{~d}$ & $52510.00 \mathrm{ab}$ & $2800.00 \mathrm{~b}$ & 19429.09 \\
\hline MoPoS+ & $181.67 f$ & $12.33 \mathrm{~g}$ & 0.07 & $0.00 \mathrm{~d}$ & $53390.00 \mathrm{a}$ & $1733.33 f$ & 16925.15 \\
\hline $\mathrm{MoP}+\mathrm{S}+$ & $1253.33 d$ & $91.00 \mathrm{c}$ & 0.07 & $0.00 \mathrm{~d}$ & $51036.67 b c$ & $2133.33 d$ & 18671.86 \\
\hline M+PoSo & $315.00 \mathrm{e}$ & $28.67 \mathrm{e}$ & 0.09 & $43.33 \mathrm{a}$ & $46026.67 d$ & $1866.67 \mathrm{e}$ & 16021.41 \\
\hline $\mathrm{M}+\mathrm{P}+\mathrm{So}$ & $1696.67 b$ & $125.00 \mathrm{~b}$ & 0.07 & $13.33 \mathrm{c}$ & $47440.00 \mathrm{~d}$ & $2433.33 c$ & 20169.61 \\
\hline $\mathrm{M}+\mathrm{PoS}+$ & $306.67 \mathrm{e}$ & $25.67 \mathrm{ef}$ & 0.08 & $46.67 \mathrm{a}$ & $38403.00 \mathrm{e}$ & $1833.33 \mathrm{e}$ & 18946.06 \\
\hline $\mathrm{M}+\mathrm{P}+\mathrm{S}+$ & $2416.67 \mathrm{a}$ & $185.00 \mathrm{a}$ & 0.08 & $33.33 b$ & $47320.00 \mathrm{~d}$ & $2966.67 a$ & 21197.80 \\
\hline LSD & 52.60 & 5.483 & 0.0554 & 7.149 & 1688 & 81.27 & 819.5 \\
\hline
\end{tabular}

* different letter implies significant differences in the same column.

Continued Table 2.

Menekşe Soil

\begin{tabular}{lllllllc} 
Treatment & $\begin{array}{l}\text { Ca content } \\
(\mathrm{mg} / \mathrm{kg})\end{array}$ & $\begin{array}{l}\text { Mg content } \\
(\mathrm{mg} / \mathrm{kg})\end{array}$ & $\begin{array}{l}\text { Fe content } \\
(\mathrm{mg} / \mathrm{kg})\end{array}$ & $\begin{array}{l}\text { Zn content } \\
(\mathrm{mg} / \mathrm{kg})\end{array}$ & $\begin{array}{l}\mathrm{Cu} \text { content } \\
(\mathrm{mg} / \mathrm{kg})\end{array}$ & $\begin{array}{l}\text { Mn content } \\
(\mathrm{mg} / \mathrm{kg})\end{array}$ & $\begin{array}{l}\text { Na content } \\
(\mathrm{mg} / \mathrm{kg})\end{array}$ \\
\hline MoPoSo & 6644.00 & $6737.13 \mathrm{~b}$ & $183.62 \mathrm{c}$ & $103.57 \mathrm{c}$ & $25.99 \mathrm{a}$ & $92.55 \mathrm{c}$ & 3314.71 \\
MoP+So & 7076.90 & $6438.90 \mathrm{c}$ & $197.08 \mathrm{~b}$ & $98.60 \mathrm{~d}$ & $18.72 \mathrm{~d}$ & $163.97 \mathrm{a}$ & 2352.79 \\
MoPoS+ & 5478.50 & $7287.73 \mathrm{a}$ & $211.17 \mathrm{a}$ & $109.17 \mathrm{~b}$ & $22.16 \mathrm{~b}$ & $137.83 \mathrm{~b}$ & 2770.13 \\
MoP+S+ & 6843.80 & $6386.33 \mathrm{c}$ & $195.44 \mathrm{~b}$ & $87.22 \mathrm{e}$ & $17.19 \mathrm{e}$ & $168.93 \mathrm{a}$ & 2072.63 \\
M+PoSo & 7043.60 & $7281.87 \mathrm{a}$ & $170.68 \mathrm{de}$ & $119.07 \mathrm{a}$ & $25.52 \mathrm{~b}$ & $94.22 \mathrm{c}$ & 2760.38 \\
$\mathrm{M}+\mathrm{P}+$ So & 6977.00 & $6353.17 \mathrm{c}$ & $167.74 \mathrm{e}$ & $87.24 \mathrm{e}$ & $14.08 \mathrm{f}$ & $88.38 \mathrm{~cd}$ & 1759.63 \\
$\mathrm{M}+\mathrm{PoS}+$ & 7243.43 & $7501.70 \mathrm{a}$ & $175.94 \mathrm{~cd}$ & $96.39 \mathrm{~d}$ & $21.27 \mathrm{c}$ & $78.40 \mathrm{e}$ & 2658.21 \\
$\mathrm{M}+\mathrm{P}+\mathrm{S}+$ & 7909.40 & $6948.23 \mathrm{~b}$ & $175.61 \mathrm{~d}$ & $89.93 \mathrm{e}$ & $17.12 \mathrm{e}$ & $82.44 \mathrm{de}$ & 2129.17 \\
$\mathrm{LSD}$ & 317.9 & 296.5 & 7.735 & 4.508 & 0.6216 & 6.475 & 157.5
\end{tabular}

\footnotetext{
* different letter implies significant differences in the same column.
} 


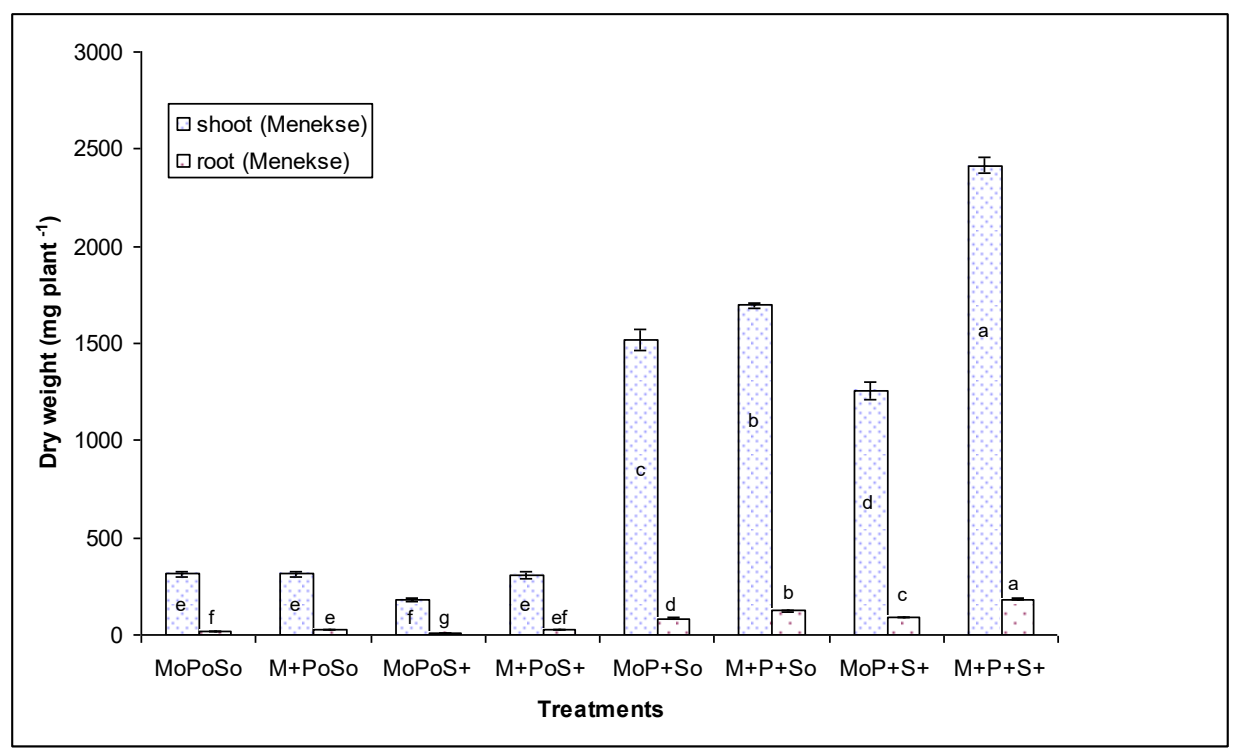

Figure 1. Pepper shoot and root dry weight following mycorrhizal inoculation and $\mathrm{P}$ and ES fertilization in Menekşe soil. Different letters indicate significant difference between the treatments. Error bars indicate standard deviation.

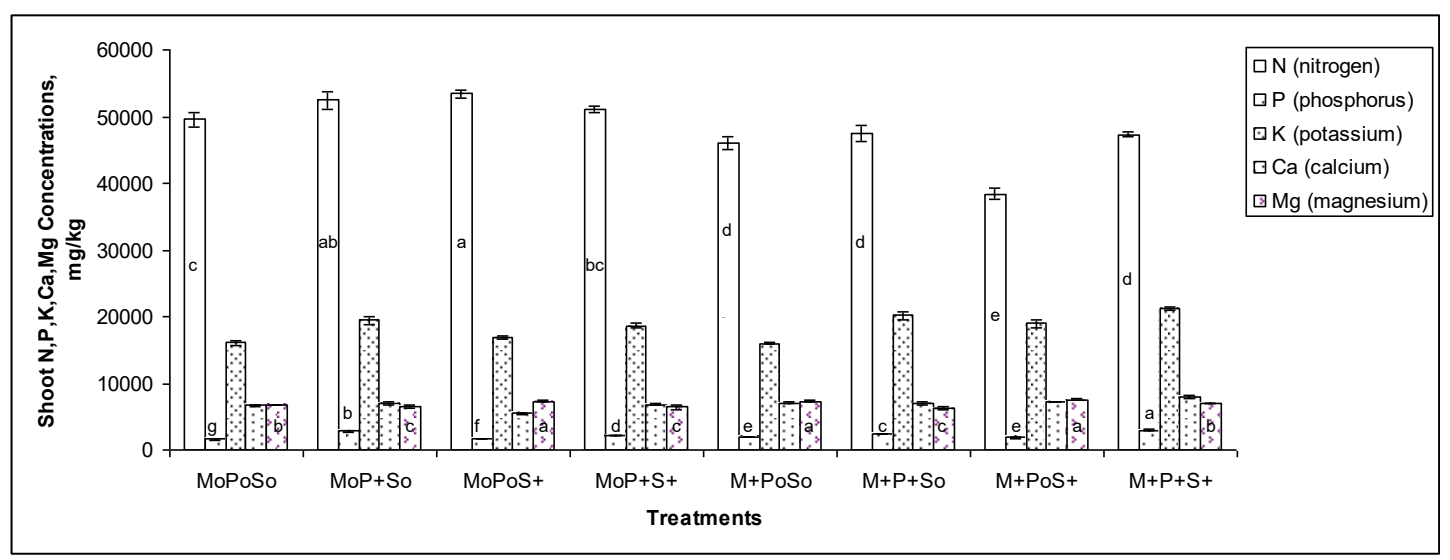

Figure 2. Pepper N,P,K,Ca,Mg content following mycorrhizal inoculation and P and ES fertilization in Menekşe soil. Different letters indicate significant difference between the treatments. Error bars indicate standard deviation.

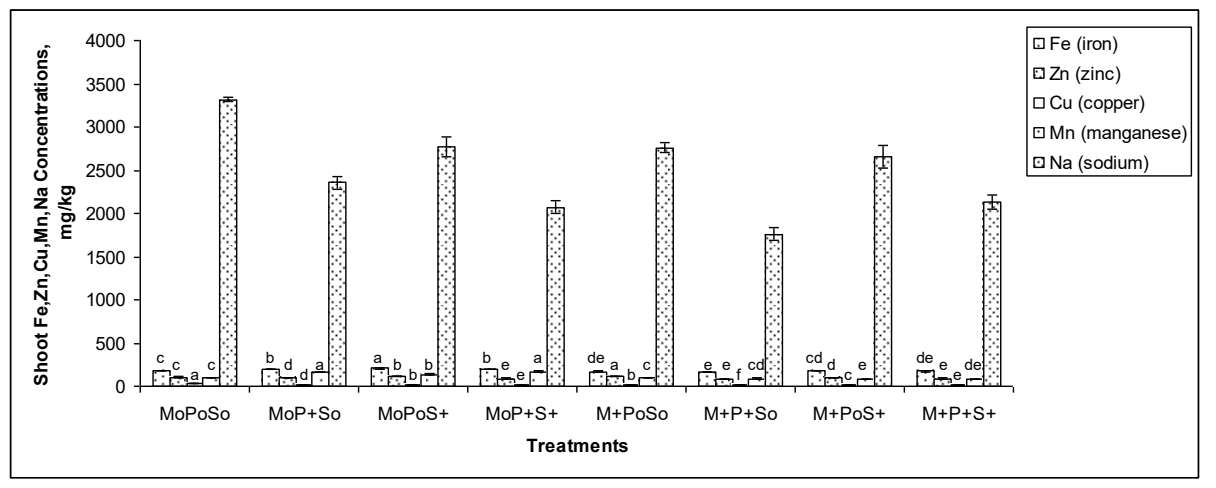

Figure 3. Pepper $\mathrm{Fe}, \mathrm{Zn}, \mathrm{Cu}, \mathrm{Mn}, \mathrm{Na}$ content following mycorrhizal inoculation and $\mathrm{P}$ and ES fertilization in Menekşe soil. Different letters indicate significant difference between the treatments. Error bars indicate standard deviation. 
$\mathrm{MoP}+\mathrm{S}+$ treatment compared to $\mathrm{MoP}+\mathrm{So}$ one significantly decreased the shoot yield whereas the root yield significantly increased in the non- mycorrhizal treatments. The shoot yield decrease can be attributed to the increased root yield with the differences in the shoot nutrient concentrations. With respect to that, while the shoot $\mathrm{Mn}, \mathrm{Mg}$ and Fe concentrations were unchanged, the shoot $\mathrm{N}, \mathrm{P}, \mathrm{Zn}$ and $\mathrm{Cu}$ concentrations were significantly lower for $\mathrm{M}_{0} \mathrm{P}+\mathrm{S}+$ treatment compared to $\mathrm{M}_{0} \mathrm{P}+\mathrm{S}_{0}$ one in the non-mycorrhizal treatments as shown in Figure 2, 3 and Table 2.

Both shoot and root yield increases were significant in the mycorrhizal treatments for $\mathrm{M}+\mathrm{P}+\mathrm{S}+$ treatment compared to $\mathrm{M}+\mathrm{P}+\mathrm{So}$ indicating the further synergism among mycorrhizae, ES and $\mathrm{P}$ compared to the synergism between mycorrhizae and phosphorus in the clay soil growth conditions. Those yield increases can also be related to the differences in the shoot nutrient concentrations, too. In that respect, the shoot $\mathrm{P}, \mathrm{Mg}, \mathrm{Fe}$ and $\mathrm{Cu}$ concentrations significantly increased while the shoot $\mathrm{Mn}$ concentration significantly decreased for the $\mathrm{M}+\mathrm{P}+\mathrm{S}+$ treatment compared to the $\mathrm{M}+\mathrm{P}+\mathrm{So}$ one with the unchanged shoot $\mathrm{Zn}$ and $\mathrm{N}$ concentrations in the mycorrhizal treatments. Moreover, $\mathrm{M}+\mathrm{P}+\mathrm{S}+$ treatment in the mycorrhizal treatments resulted in the highest yield where the highest shoot nutrient concentration was P among the all treatments. On the other hand, those differences in the yield and shoot nutrient concentrations are reciprocally culminated in for the response to the root morphological changes (Figure 4).

Those results above indicate that different treatments in clay soil growth conditions affect the efficient use of nutrients and mycorrhizae with the subsequent root morphological changes in plant resulting in those yield differences.
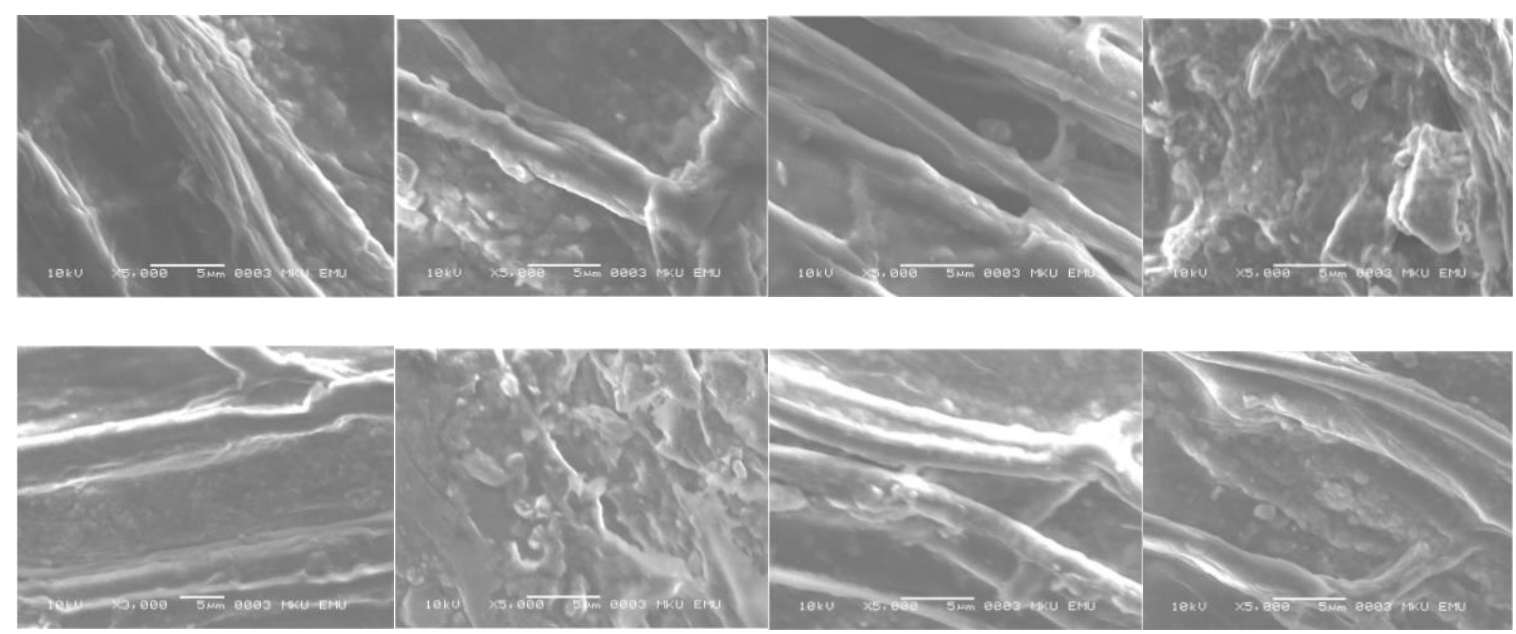

Figure 4. Root morphologies of Menekşe soil from the left to the right for the response of the treatments of MoPoSo, MoPoS+, MoP+So, MoP+S+ above, and M+PoSo, M+PoS+, M+P+So, M+P+S+ below, respectively.

\section{Root Mycorrhizal Infection Responses to ES, P and Mycorrhizal Inoculation}

The highest root mycorrhizal infection levels in the mycorrhizal treatments by $\mathrm{M}+\mathrm{PoSo}$ and $\mathrm{M}+\mathrm{P}_{0} \mathrm{~S}+$ treatments without $\mathrm{P}$ fertilization were obtained. However, $\mathrm{M}+\mathrm{P}+\mathrm{So}$ or $\mathrm{M}+\mathrm{P}+\mathrm{S}+$ treatment compared to $\mathrm{M}+\mathrm{PoSo}$ and $\mathrm{M}+\mathrm{P}_{0} \mathrm{~S}+$ ones in the mycorrhizal treatments significantly decreased the root mycorrhizal infection level. Those findings are in line with the previous findings (Baylis, 1967; 
Mosse, 1967; Karaca, 2012a) who reported that mycorrhizal infections tend to stop in soils containing or given high $\mathrm{P}$. However, $\mathrm{M}+\mathrm{PoS}+$ treatment compared to $\mathrm{M}+\mathrm{PoSo}$ one in the mycorrhizal treatments did not affect the root mycorrhizal infection level as shown in Figure 5. Those findings are not consistent with the previous findings (Diaz et al., 1996) who reported that a slight reduction on percentage of mycorrhizal colonization was noted by $\mathrm{SO}_{2}$ treatment. On the other hand, the ES addition compensated the decreasing effect of $\mathrm{P}$ fertilization to some extent in the root mycorrhizal infection level being in line with the previous findings (Karaca, 2012a). Moreover, $\mathrm{M}+\mathrm{P}+\mathrm{S}+$ treatment compared to the $\mathrm{M}+\mathrm{P}+\mathrm{So}$ treatment in the mycorrhizal treatments resulted in the highest root and shoot yields among the all treatments (Table 2). This clearly shows that ES increases the efficient work of mycorrhizae beside compensation of the decreasing effect of $\mathrm{P}$ in the root mycorrhizal infection level. Those changing root infection levels can be related to the different treatments in the plant growth conditions.

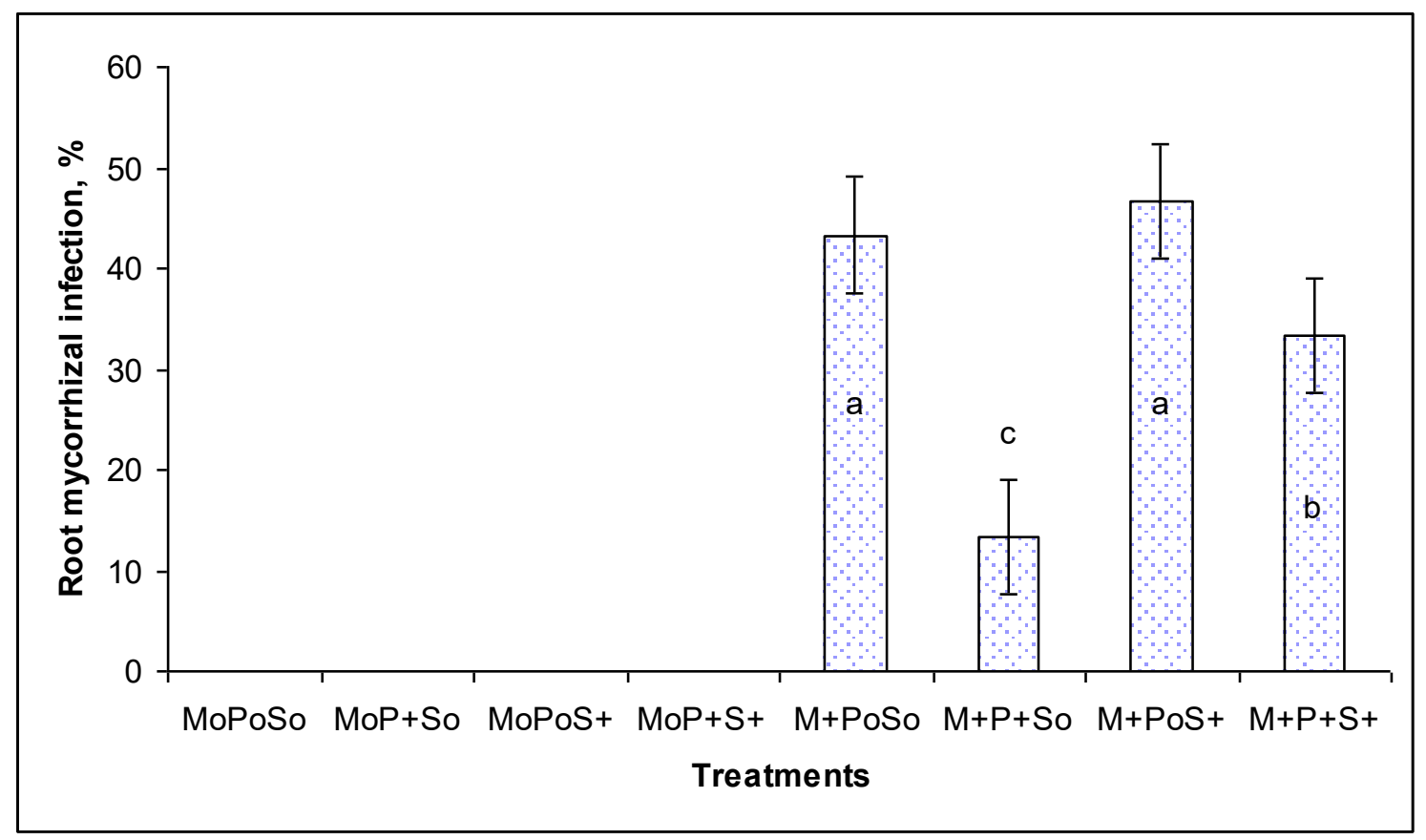

Figure 5. Pepper mycorrhizal infection percent following mycorrhizal inoculation and $\mathrm{P}$ and ES fertilization in Menekşe soil. Different letters indicate significant difference between the treatments. Error bars indicate standard deviation.

\section{Root to Shoot ratio}

$\mathrm{M}+\mathrm{PoSo}$ treatment compared to the MoPoSo one did not affect the root to shoot ratio. Similarly, the root to shoot ratio were statistically unchanged among the all treatments including mycorrhizal and non-mycorrhizal ones. Those indifferences in the root to shoot ratio are similar to the previous findings (Ortas et al., 2002; Karaca, 2012a). Eventhough, statistically insignificant fluctuating root to shoot ratios among the treatments came out (Table 1), there were no correlations all the time between the yield level and root to shoot ratio for the different treatments in the growth medium conditions. Accordingly, higher yield compared to lower yield may have the root to shoot ratio trend in the both directions as presented in Figure 6. Those findings are similar to the findings (Karaca, 2012a). Those ratios may lend support to the hypothesis (Romero et al., 1996) who proposed that there may be an optimum root to shoot ratio for plant growth. 


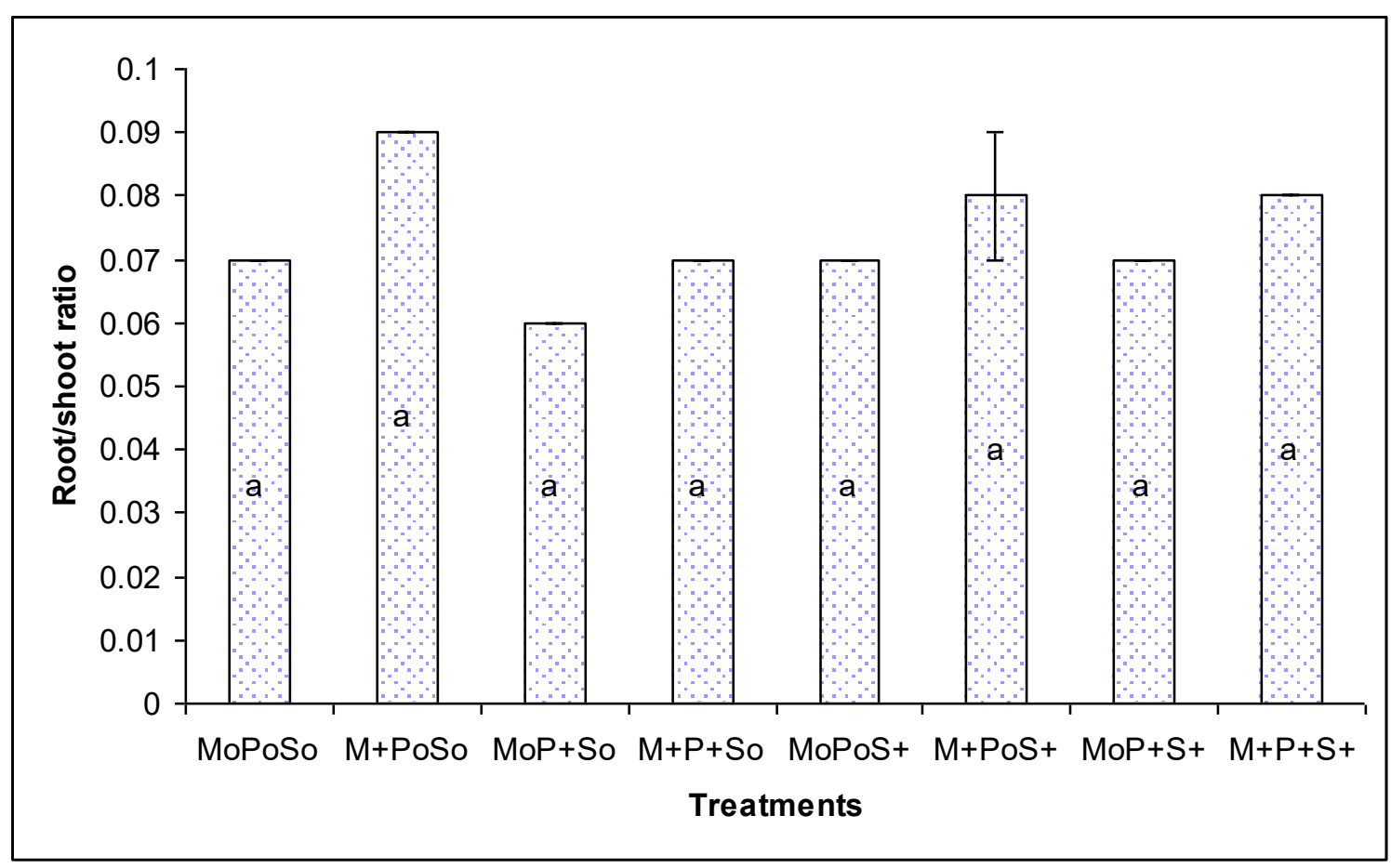

Figure 6. Pepper root to shoot ratio (dry weight) following mycorrhizal inoculation and P and ES fertilization in Menekşe soil. Different letters indicate significant difference between the treatments. Error bars indicate standard deviation.

\section{DISCUSSION}

Mycorrhizal inoculation alone compared to control treatment resulted in higher root yield with unchanged shoot yield. That indicates that the root growth has the priority for plant growth to be able to create investment potential for the future shoot growth depending upon the growth period of plant. Concomitantly, the shoot $\mathrm{N}, \mathrm{P}, \mathrm{K}, \mathrm{Ca}, \mathrm{Mg}, \mathrm{Fe}, \mathrm{Mn}, \mathrm{Zn}, \mathrm{Cu}$ and $\mathrm{Na}$ concentrations were diluted, accumulated or unchanged for any nutrient independent from each other as the yield response. However, $\mathrm{P}$ fertilization and mycorrhizal inoculation in combination compared to the $\mathrm{P}$ fertilization alone created mutual stimulative effect resulting in the significantly increased shoot and root yield. That shows that the growth of pepper in the clay soil growth conditions is vigorous as a response to the $\mathrm{P}$ fertilization in the mycorrhizal treatments. On the other hand, the increase of shoot and root yield by the $\mathrm{P}$ fertilization in the mycorrhizal treatments increased significantly farther by ES and $\mathrm{P}$ fertilization in combination resulting in the highest yields among the all treatments. In relation to that, the changed shoot nutrient concentrations in both direction for any nutrient independent from any other one seems to be also related to the subsequent yield differences in the plant growth conditions. Consequently, soil type, mycorrhizae inoculation, treatment and plant growth period can be involved in creating the further yield differences. It can be emphasized that fertilization regulations in clay soil growth conditions can be related to increase the efficient work of mycorrhizae for the vigorous growth of pepper to some great extent.

The increased efficient work of mycorrhizae resulting in the highest shoot and root yield in the clay soil growth conditions by ES and P fertilization in combination for pepper may shed light to prevent yield losses resulting from heavy metal accumulation in plant tissues to obtain higher yield in agriculture. Accordingly, regulation of fertilizer forms and doses can lead to contribution of the plant production system causing the efficient work of mycorrhizae. 


\section{Acknowledgments}

This project was partially funded by Cukurova University Research Foundation. Appreciation is extended to the workers of Institute of Applied Sciences, Mustafa Kemal University for their help in digesting and analyzing plant samples.

\section{References}

Baylis, G.T.S. 1967. Experiments on the ecological significance of phycomycetous mycorrhizas. New Phytologist 66:231.

Blake, G.R. and K.H. Hartge. 1986a. Bulk density. In Methods of Soil Analysis. Part 1. Physical and mineralogical methods, ed. A. Klute (2 ${ }^{\text {nd }}$ Edn.), pp.363-375. Madison, WI: SSSA Book Series 5.

Blake, G.R. and K.H. Hartge. 1986b. Particle density. In Methods of Soil Analysis. Part 1. Physical and mineralogical methods, ed. A. Klute ( $2^{\text {nd }}$ Edn.), pp.377-382. Madison, WI: SSSA Book Series 5.

Bouyoucos, G.J. 1951. A recalibration of the hydrometer method for making mechanical analysis of soils. Agronomy Journal 43:434-438.

Bremner, J.M. 1996. Nitrogen-total. In: Method of Soil Analysis. Part 3. Chemical methods, eds. D.L Sparks, A.L. Page, P.A. Helmke, R.H. Loeppert, P.N. Soltanpour, M.A. Tabatabai, C.T. Johnston, and M.E. Sumner, pp.1085-1122. Madison, WI: SSSA.

Cui, Y., Y. Dong, H. Li, and Q. Wang. 2004. Effect of elemental sulfur on solubility of soil heavy metals and their uptake by maize. Environment International. 30 (3): 323-328.

Diaz, G., O. Barrantes, M. Honrubia, and C. Gracia. 1996. Effect of ozone and sulfurdioxide on mycorrhizae of Pinus halepensis miller. Annales des Sciences Forestieres 53: 849-856.

Fabig, W., J.C.G. Ottow, and F. Muller. 1978. Mineralisation von 14C-Markiertem Benzoat Mit Nitrat als Wasser-Stoffakzeptor unter Vollstandig Anaeroben Bedingungen Sowie Bei Vermindertem Sauerstoffpartialdruck [Mineralization of 14C-labeled benzoate with nitrate as water under completely anaerobic conditions, as well as partially heavy clay oxygen]. Landwirstschaft Forschung, 35: 441-453.

Fachgruppe Wasserchemie in der Gesellscheft Deutscher Chemiker. 1983. Deutsche Einheitsverfahren Zur Wasser-Abwasser-und Schlammuntersuchungen [German Standart Methods for Water, Wastewater and Sludge Tests]. Weinheim: Verlag Chemie.

Gerdemann, J.W. and T.H. Nicolson. 1963. Spors of mycorrhizal endogeny species extracted from soil by wet sieving and decanting. Transactions of the British Mycological Society 46: 235-244.

Guo, T., J.L. Zhang, and X.L. Li. 2005. Effect of sulfur supply and mycorrhizal colonization on the growth and pungency of Allium cepa L. In: Plant Nutrition for Food Security, Human Health and Environmental Protection, ed.C.J.Li, pp. 860-861. Tsinghua, China: Tsinghua University Press.

Hoffman, C., H.J. Koch, G. Schlinker, G. Sander, M. Saurer, and K. Burcky. 1998. Supply and nutrient demand of sugar beet for sulphur. Zuckerindustrie 123: 675-682.

Kacar, B. 1972. Chemical analysis of soil and plant (in Turkish). Ankara, Turkey: Ankara University Agricultural Press 453.

Karaca, H. 2012a. Buffering effect of elemental sulfur on mycorrhizal infection of leek. Journal of Plant Nutrition 35(5): 678-687.

Karaca, H. 2012b. Efficiency of elemental sulfur on mycorrhizae in the yield increase of wheat. Journal of Plant Nutrition 35(8): 1225-1233.

Karaca, H. 2013. Effects of elemental sulfur and mycorrhizae on the yield of wheat in different soils. Journal of Plant Nutrition. 
Karaca, H., V. Uygur, A. Özkan, and Z. Kaya. 2013. Effects of mycorrhizae and fertilization on soybean yield and nutrient uptake. Communications in Soil Science and Plant Analysis. 44(16): 2459-2471.

Klute, A. and C. Dirksen. 1986. Hydraulic Conductivity and Diffusivity: Laboratory Methods. In Methods of Soil Analysis. Part 1. Physical and mineralogical methods, ed, A. Klute (2 ${ }^{\text {nd }}$ Eds.). Madison, WI: SSSA Book Series 5.

Koske, R.E. and J.N.Gemma. 1989. A modified procedure for staining roots to detect VAM. Mycological Research 92: 486-505.

Lees, R. 1971. Laboratory Handbook of Methods of Food Analysis. London: Leonard Hill Books.

Lindsay, W.L. and W.A. Norwell. 1978. Development of DTPA for soil test zinc, iron, manganese and copper. Soil Science Society of America Journal 42: 421-428.

Loeppert, R.H. and D.L. Suarez, 1996. Carbonate and gypsum. In: Method of soil analysis. Part 3. Chemical methods, eds, D.L Sarks, A.L. Page, P.A. Helmke, R.H. Loeppert, P.N. Soltanpour, M.A. Tabatabai, C.T. Johnston, and M.E. Sumner, pp. 437-474. Madison, WI: SSSA.

McLaughlin, B.D. and I.C.R. Holford. 1982. Initial and medium -term responses of white clover to three sulfur fertilizers on a basaltic soil. Australian Journal of Experimental Agriculture and Animal Husbandry 22(115): 9599.

Menge, J.A., C.K. Labanuskas, E.L.V. Johnson, and R.G. Platt. 1978. Partial substitution of mycorrhizal fungi for phosphorous fertilization in the greenhouse culture of citrus. Soil Science Society of American Journal 42 : 926-930.

Merrien, A. 1987. La fertilization soufree du colza: un oubli qui peut couter cher! Perspectives Agricoles 115:201-203.

Mosse, B. 1967. Effects of host nutrient status on mycorrhizal infection. Annual Report of the Rothamsted Experiment Station, p.79.

Murphy, J. and J.P. Riley. 1962. A modified single solution method for the determination of phosphate in natural waters. Analytica Chimica Acta 27:31-36.

Olsen, S.R., C.V. Cole, F.S. Watanabe, and L.A. Dean. 1954. Estimation of available phosphorus in soils by extraction with sodium bicarbonate. USDA Circular 939. Washington, DC: US Government Printing Office.

Ortas, I., P.J. Harris, and D.L. Rowell. 1996. Enhanced uptake of phosphorous by mycorrhizal sorghum plants as influenced by forms of nitrogen. Plant and Soil 184: 255-264.

Ortas, I., D. Ortakc1, and Z. Kaya. 2002. Various mycorrhizal fungi propagated on different hosts have different effect on citrus growth and nutrient uptake. Communications in Soil Science and Plant Analysis 33(1\&2): 259272.

Ortas, I., D. L. Rowell, and P.J. Haris. 2004. Effect of mycorrhizae and pH change at the root soil interface on phosphorus uptake by sorghum using a rhizocyclinder technique. Communications in Soil Science and Plant Analysis 35: 1061-1080.

Özbek, H., U. Dinç, and S. Kapur. 1974. Detailed basic survey and mapping of the soils of Cukurova University settlement area (in Turkish). Cukurova University Agricultural Faculty publication 73. Adana, Turkey: Cukurova University.

Pratt, P.F. and H.H. Morse. 1954. Potassium release from exchangeable and non-exchangeable forms in Ohio soils. Ohio Agricultural Experiment Station Research Bulletin 747. Columbus, OH: Ohio State University.

Raj, J., D.J, Bagyaraj, and A. Manjunath. 1981. Influence of soil inoculation with vesicular-arbuscular mycorrhizae and a phosphate-dissolving bacterium on plant growth and ${ }^{32} \mathrm{p}$ uptake. Soil Biology and Biochemistry 13(2): 105-108. 
Romero, A., J. Ryder, J.T. Fisher, and J.G. Mexal. 1986. Root system modification of container stock for arid land plantings. Forest Ecology and Management 16: 281-290.

Schlichting, E. and H. Blume. 1966. Bodenkundliches Practikum [Soil Science Handbook].Berlin: Paul Parey Verlag.

U.S. Salinity Laboratory Staff. 1954. Diagnosis and improvement of saline and alkali soils. USDA Handbook 60. Washington, DC: U.S. Government Printing Office.

Yibirin, H., J.W. Johnson, and D. Eckert. 1996. Corn production as affected by daily fertilization with ammonium, nitrate, and phosphorous. Soil Science Society of America Journal 60: 512-518. 